\title{
BMJ Open Real-world challenges for patients with breast cancer in sub-Saharan Africa: a retrospective observational study of access to care in Ghana, Kenya and Nigeria
}

\author{
Majid Twahir (D) , ${ }^{1}$ Razaq Oyesegun, ${ }^{2}$ Joel Yarney, ${ }^{3}$ Andrew Gachii, ${ }^{4}$ \\ Clement Edusa, ${ }^{5}$ Chukwumere Nwogu, ${ }^{6}$ Gitangu Mangutha, ${ }^{7}$ Philip Anderson, ${ }^{8}$ \\ Emmanuel Benjamin, ${ }^{9}$ Borna Müller, ${ }^{10}$ Charles Ngoh $^{7}$
}

To cite: Twahir M, Oyesegun $\mathrm{R}$, Yarney J, et al. Real-world challenges for patients with breast cancer in sub-Saharan Africa: a retrospective observational study of access to care in Ghana, Kenya and Nigeria. BMJ Open 2021;11:e041900. doi:10.1136/ bmjopen-2020-041900

- Prepublication history is published online only. To view please visit the journal online (http://dx.doi.org/10.1136/ bmjopen-2020-041900).

Received 22 June 2020 Revised 05 February 2021 Accepted 10 February 2021

Check for updates

(C) Author(s) (or their employer(s)) 2021. Re-use permitted under CC BY-NC. No commercial re-use. See rights and permissions. Published by BMJ.

For numbered affiliations see end of article.

Correspondence to

Dr Majid Twahir;

mtwahir@gmail.com

\section{ABSTRACT}

Objective To evaluate medical resource utilisation and timeliness of access to specific aspects of a standard care pathway for breast cancer at tertiary centres in subSaharan Africa.

Design Data were retrospectively abstracted from records of patients with breast cancer treated within a prespecified 2-year period between 2014 and 2017. The study protocol was approved by local institutional review boards.

Setting Six tertiary care institutions in Ghana, Kenya and Nigeria were included.

Participants Health records of 862 patients with breast cancer were analysed: 299 in Ghana; 314 in Kenya; and 249 in Nigeria.

Interventions As directed by the treating physician. Outcome measures Parameters selected for evaluation included healthcare resource and use, medical procedure turnaround times and out-of-pocket (0OP) payment patterns.

Results Use of mammography or breast ultrasonography was $<45 \%$ in all three countries. Across the three countries, $78 \%-88 \%$ of patients completed tests for hormone receptors and human epidermal growth factor receptor 2 (HER2). Most patients underwent mastectomy $(64 \%-67 \%)$ or breast-conserving surgery $(15 \%-26 \%)$. Turnaround times for key procedures, such as pathology, surgery and systemic therapy, ranged from 1 to 5 months. In Ghana and Nigeria, most patients (87\%-93\%) paid for diagnostic tests entirely 00P versus $30 \%-32 \%$ in Kenya. Similarly, proportions of patients paying 00P only for treatments were high: $45 \%-79 \%$ in Ghana, $8 \%-20 \%$ in Kenya and $72 \%-89 \%$ in Nigeria. Among patients receiving HER2-targeted therapy, the average number of cycles was five for those paying 00P only versus 14 for those with some insurance coverage.

Conclusions Patients with breast cancer treated in tertiary facilities in sub-Saharan Africa lack access to timely diagnosis and modern systemic therapies. Most patients in Ghana and Nigeria bore the full cost of their healthcare and were more likely to be employed and have secondary or postsecondary education. Access to
Strengths and limitations of this study

- A key strength of this study was that it included a real-world dataset from a relatively large sample of patients (>800) from sub-Saharan Africa.

- In addition, the study included data from both private-run and government-run tertiary care centres.

- The study collected and reports patient-level geocoded data.

- A key study limitation was missing data relating to disease stage and outcomes.

screening/diagnosis and appropriate care is likely to be substantively lower for the general population.

\section{INTRODUCTION}

In sub-Saharan Africa, breast cancer is one of the most frequently diagnosed malignancies and one of the most common causes of cancer-related death. ${ }^{1-3}$ Socioeconomic factors, including delays in initiating standard treatments or procedures because of lack of patient education, limitations in availability of or access to care and financial burden are key resource-related factors that compound the challenge of managing breast cancer in this region. ${ }^{4}$ Consequently, the agestandardised mortality rates among patients with breast cancer in Africa are among the highest in the world, especially in North and West Africa with age-standardised mortality rates of 18.4 and 17.8 per 100 000, respectively. ${ }^{16}$ Established patient risk factors, such as age, sex, age at onset of menarche, age at first full-term pregnancy, breast feeding, age at onset of menopause, obesity and physical activity are trending towards increased 
incidence of breast cancer in Africa. ${ }^{7}$ These findings are aligned with epidemiological extrapolations suggesting that the relative proportion of the breast cancer at-risk population is steadily increasing in sub-Saharan African countries, such as Nigeria, at approximately $2.5 \%$ annually $^{7}$; this contrasts with developed European countries, such as France, in which this segment of the population has plateaued. Taken together, these observations suggest that the number of breast cancer related deaths in sub-Saharan Africa will continue to increase in the coming decade and that effective allocation of financial and healthcare resources for the management of breast cancer is an important goal.

Identifying barriers to treatment and resource limitations in the context of specific aspects of standard care along a patient's journey is a key first step to provide insights to reform healthcare policy, infrastructure and resources to improve access and affordability of care and subsequent outcomes for patients with breast cancer. Accordingly, we collected data on resource utilisation, turnaround times for clinical procedures, treatment, financial support for various aspects of care and demographics for patients with a diagnosis of breast cancer who received care at tertiary cancer centres in three subSaharan African countries: Ghana, Kenya and Nigeria. The countries were selected for their relatively developed healthcare infrastructure compared with most countries in the region, and for each country, we included one private and one government-run cancer centre to cover any potential differences in practice, access or cost burden between these types of institutions. In the current report, we summarise access to care and out-of-pocket (OOP) payment patterns and attempt to delineate the challenges and limitations faced by patients with breast cancer in sub-Saharan Africa.

\section{METHODS}

\section{Study design}

The aim of the study was to systematically evaluate medical resource utilisation data. In addition, the time intervals to access key recommended interventions for individual patients over the course of their treatment journey since diagnosis in government-operated or privately operated tertiary cancer centres were extracted.

This analysis was based on a retrospective data review of patients with a diagnosis of breast cancer who were treated at tertiary cancer centres in three sub-Saharan African countries: Ghana, Kenya and Nigeria. Within each country, patient records were obtained from two tertiary institutions, one government operated and the other privately operated. The study sites were as follows: Ghana: Korle-Bu Teaching Hospital and Sweden Ghana Medical Centre; Kenya: Kenyatta National Hospital and The Aga Khan University Hospital; and Nigeria: National Hospital Abuja and Lakeshore Cancer Centre. The study protocol was approved by the local institutional review boards, all of which deemed that patient informed consent was not necessary as all patient data were de-identified.

Patients with breast cancer who initiated their treatment journey from definitive diagnosis through to treatment at one of the aforementioned institutions during the study period and who completed the core part of assessment (diagnosis) and treatment were included in the analysis. Patients presenting at these tertiary cancer centres from 2014 to 2016 (Nigeria and Ghana) or from 2015 to 2017 (Kenya) were included. All available data from patient charts were extracted until the patient's loss to follow-up, death or completion of the study.

\section{Data collection}

All available patient chart data were extracted using a structured data collection tool that was deployed as a mobile application. Data integrity was assured through quality checks in real time, facilitated by two-way interaction between the healthcare professionals involved in abstracting the patient-record data onsite and a central data collection team. The digital framework of the data collection application allowed for the necessary synchronisation of inputs and revisions to the collated data, the intermittent saving of input and the use of offline data entry modes. Healthcare professionals involved in data collection had digital access to the standard operating procedures for data collection through their mobile devices to address procedural and data definition queries. In addition to the initial demographic information, which included employment and education status, disease stage (per the American Joint Committee on Cancer Staging Manual, 7 th edition) ${ }^{8}$ and other key variables (ie, patient's age, level of education, occupation, number of facilities visited, presenting complaints and duration of initial symptoms), data relating to the following key areas were extracted: medical history and initial symptoms, primary laboratory investigations, diagnostic imaging, histopathology, immunohistochemistry, treatments (ie, primary interventions and supportive care), admission care, follow-up, and cost and payment information. Information relating to dates at which diagnostic tests were recommended and dates at which test results became available, as well as dates for treatment recommendation and treatment initiation, were extracted from patient records and used to estimate turnaround times.

\section{Statistical analyses}

This was an observational study, and no formal hypothesis was tested. All data were summarised descriptively. Continuous variables were summarised using means and SD, and categorical variables were summarised using percentages. Summary analyses included baseline demographic and disease characteristics, tests and procedures performed, turnaround time (time to completion of specific tests and procedures from defined events in patient journey (eg, interval from presenting to biopsy and biopsy to pathology)), payment patterns and costs. All parameters were summarised by country and type of 
institution. Summary statistics were performed in R statistical software (The R Foundation) and Microsoft Excel 2016 (Microsoft, Redmond, Washington, USA).

\section{Patient and public involvement}

There was no patient involvement at any stage of the study.

\section{RESULTS \\ Patients}

This study was based on the health records of 862 patients with breast cancer, of whom 299 (34.7\%) were treated in Ghana, 314 (36.4\%) in Kenya and 249 (28.9\%) in Nigeria. The mean age of patients at diagnosis was approximately 49 years across all three countries, and most patients were employed and had been referred to the tertiary centre (table 1). Among patients with available data, those in Ghana and Nigeria were more likely to have postsecondary education compared with those in Kenya. In both Ghana and Kenya, most patients $(\sim 62 \%$ in both countries) had advanced disease (stage III/IV) at diagnosis compared with Nigeria, where most patients $(55 \%)$ had early stage (stage I/II) disease. The mean (range) commute to a tertiary cancer centre was $45 \mathrm{~km}$ $(1-614 \mathrm{~km})$ in Ghana, $56 \mathrm{~km}(2-398 \mathrm{~km})$ in Kenya and $121 \mathrm{~km}(1-717 \mathrm{~km})$ in Nigeria. Geocoded data showing individual patient geographic location by stage are available online at https:/ / bit.ly/BCPJ-Geocoded.

\section{Institutions}

The institutional resources available during the 2-year period of the patient journey are provided in table 2 . The most notable observation was the complete absence of resources to provide radiation therapy for patients with breast cancer in Nigeria during the study period.

\section{Access to care}

Health services

The use of core needle biopsy was $76 \%$ in Kenya and Nigeria but only $50 \%$ in Ghana. The relatively lower proportion of core needle biopsy recorded in Ghana was an artefact of the recording process, as many patients had biopsies performed at facilities other than the tertiary centres at which the tissues were tested. Across all three countries, $91 \%-98 \%$ of patients completed blood count/ chemistry and more than $75 \%$ of patients completed immunohistochemistry tests for hormone receptor and human epidermal growth factor receptor 2 (HER2) (figure 1).

The use of common imaging modalities (eg, mammography and breast ultrasonography) was less than $45 \%$ in all three countries. The extent of disease involvement was most ascertained using X-ray methodology. For example, chest X-ray was the most commonly used imaging methodology in Ghana (63\%) and Nigeria (61\%), whereas CT scan of the chest was the predominant methodology in Kenya (55\%) compared with Ghana (36\%) and Nigeria
$(37 \%)$. A similar trend was observed for imaging of the abdominal region to evaluate the presence of metastases.

\section{Treatment}

Almost all patients underwent surgery, with most (64\%$67 \%$ ) undergoing mastectomy and a smaller proportion (15\%-26\%) undergoing breast-conserving surgery. Access to and utilisation of radiotherapy was highly variable between countries, with $81 \%$ of patients in Ghana undergoing radiotherapy compared with only $54 \%$ and $28 \%$ of patients in Kenya and Nigeria, respectively. The use of newer generation hormonal agents, such as aromatase inhibitors, was low (21\%-29\%). In addition, the proportion of patients received HER2-targeted therapy $(5 \%-8 \%)$. In general, while most patients had access to surgery during their treatment journey, the proportion of patients accessing systemic therapies is relatively less in all three countries.

In a separate analysis among the subset of patients receiving HER2-targeted therapy, the average number of cycles was five for patients paying OOP only compared with 14 for patients with some level of healthcare insurance coverage (figure 2).

\section{Timeliness of care}

The median procedure turnaround time in the early phase of the patient journey (ie, biopsy and pathology) was highest in Ghana, where it exceeded 7 months (figure 3). This may be due in part to the extensive use of neoadjuvant chemotherapy of six cycles prior to mastectomy and definitive pathology reporting. The study is silent on the use of wide local excision for diagnosis that will provide pathological details to enable initiation of cancer therapies. Although the time to complete biopsy and pathology was lower in Kenya and Nigeria, the median exceeded 2 months in both countries. The median turnaround time for surgery was also exceedingly high in Ghana (3 months in the government setting and 5 months in the private setting) and in the government setting for Nigeria ( $\sim 4$ months). This may also be due in part to the use of neoadjuvant chemotherapy in Ghana. In all other centres, the median turnaround time for surgery was 2-6 weeks. In contrast, patients in Ghana could more readily access systemic interventions (median of 2 months in the government setting and 2 weeks in the private setting) compared with a median of 3-5 months in any setting in Kenya and Nigeria.

No clear systematic differences in procedure turnaround times between government-run and privately-run tertiary care centres could be ascertained.

\section{Payment/insurance}

Almost all patients in Ghana and Nigeria (87\%-93\%) paid for their diagnostic tests entirely OOP compared with 30\%-32\% in Kenya (figure 4). Similar to diagnostic testing, the proportion of patients paying OOP only for treatments was high: $72 \%-89 \%$ in Nigeria, $45 \%-79 \%$ in Ghana and 8\%-20\% in Kenya. Compared with Ghana 


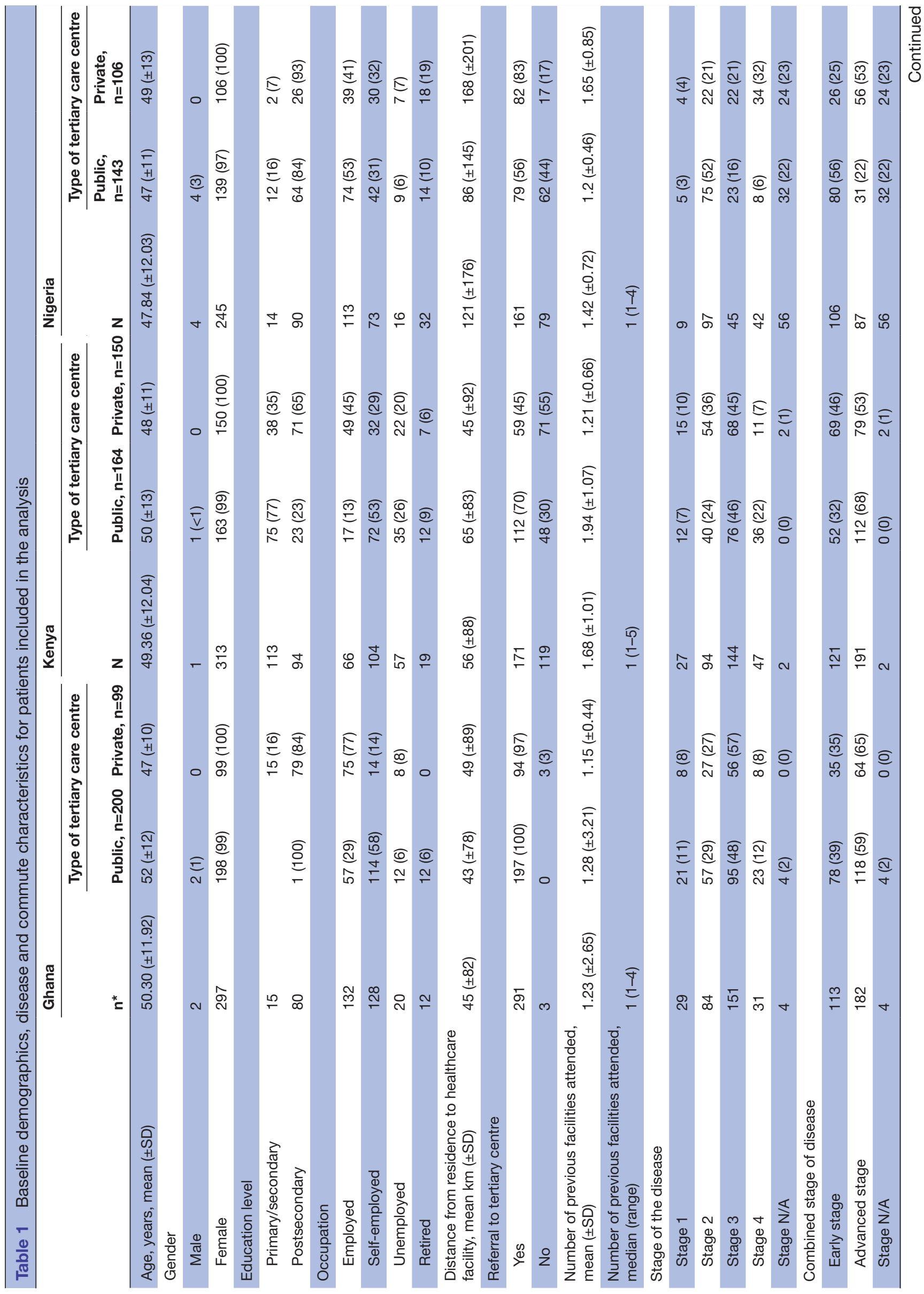


and Nigeria, a greater proportion of patients in Kenya received some sort of financial support for their treatment and procedures.

\section{DISCUSSION}

This real-world study provides insights into healthcare resource access and the OOP financial burden of breast cancer care from both the patient and institutional perspective. Doctors in tertiary facilities mostly recommended standard diagnostic procedures. The collated study data show that access to or use of common imaging modalities, such as a diagnostic mammography or breast ultrasonography, in Ghana was lower than that observed in developed economies, such as the USA, although the rates of access in Kenya and Nigeria were comparable. ${ }^{9}$ It is unclear whether the low rates of access to common imaging modalities observed in Ghana are due to resource constraints, lack of patient education or presentation of disease at late stage; however, previous studies have reported that it may be a combination of these factors. ${ }^{4510}$ The use of core needle biopsy and access to immunohistochemistry testing in these three sub-Saharan countries was also relatively similar to the rates observed in developed countries. ${ }^{1112}$ The high rate of utilisation of immunohistochemistry tests observed in this study may be due to the fact that these patients were being treated at tertiary cancer centres rather than in local/community practice. Thus, for the most part, it appears that the rate of utilisation of key diagnostic procedures required for treatment decision making for managing breast cancer in Ghana, Kenya and Nigeria in the tertiary cancer centres included in this study compares favourably with the realworld data from developed nations.

As mentioned, however, survival outcomes for patients with breast cancer in African countries remain among the poorest in the world. It is clear that the overall procedure turnaround times in Ghana, Kenya and Nigeria were much longer than those documented in developed countries. ${ }^{13}{ }^{14}$ For example, a recent analysis of Medicare patients in the USA reported median turnaround times ranging from 14 to 17 days for abnormal screening mammogram to biopsy, 39-52 days for mammogram to treatment and 20-27 days from biopsy to treatment. ${ }^{13}$ Although the Medicare metrics are evaluated and defined slightly differently than those reported in this study, the observed turnaround times for biopsy or immunohistochemistry, diagnostic imaging and initial surgery observed in this study could be extrapolated to an interval between presentation and treatment ranging from approximately 6 months to 1 year. Thus, there is a significant delay in the initiation of breast cancer treatment following initial presentation in these sub-Saharan countries, and this has a potentially adverse impact on outcomes. Possible reasons for this delay may be that the locations of tertiary care centres are widely dispersed with no clear referral infrastructure between them or that patients have financial constraints and require time to arrange for funds for 
Table 2 Institutional resources during the 2-year period of the patient journey

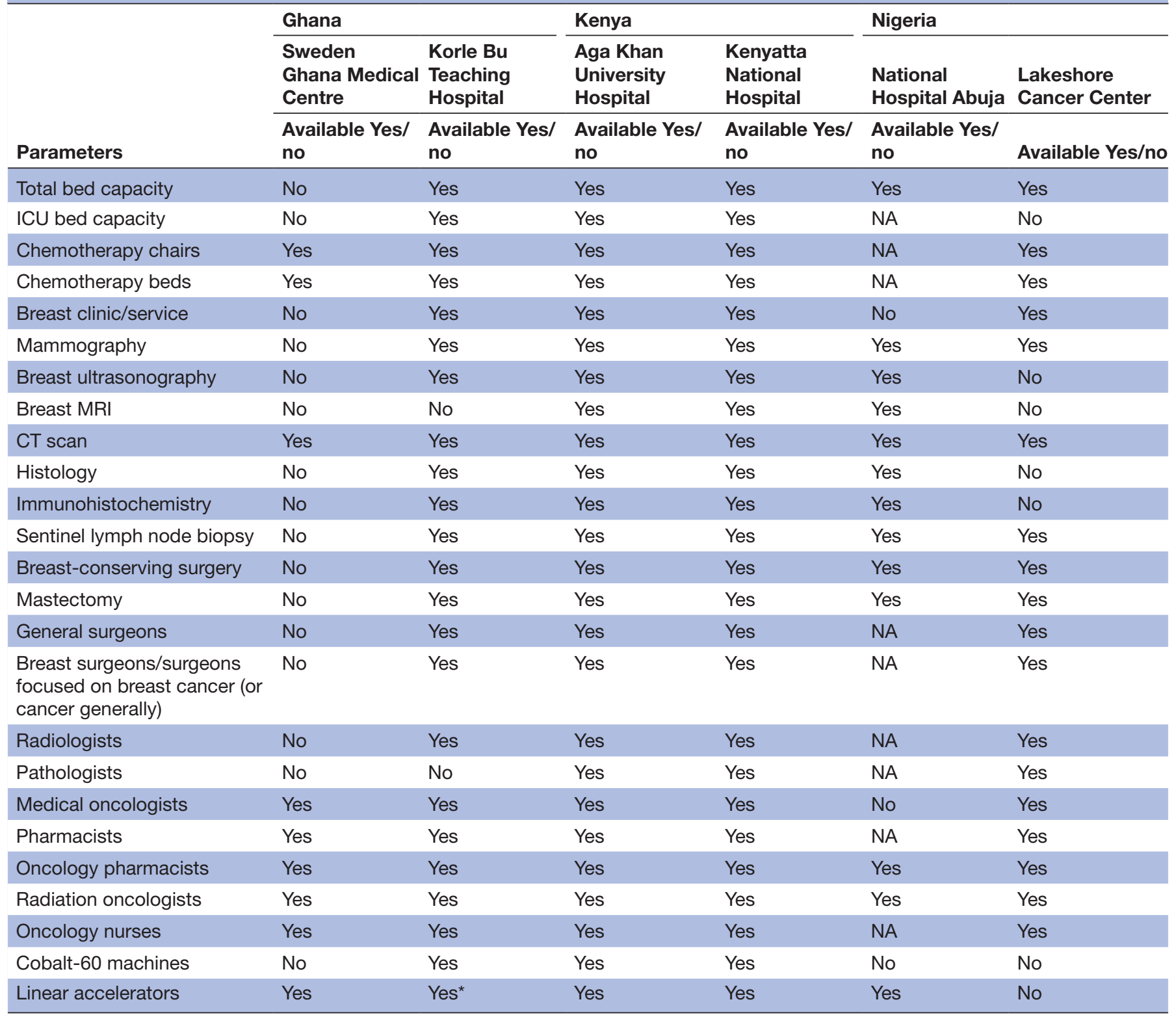

'NA'=information not available for the institution at the time of data collection.

'Yes'=present at the institution at the time of data collection

'No'=not present at the institution at the time of data collection.

*Not yet commissioned.

ICU, intensive care unit.;

the procedures or interventions. Notably, much shorter turnaround times for the initial diagnostic procedures for breast cancer management have been reported from Botswana, ${ }^{15}$ which is one of the most developed African countries.

Most patients in Ghana and Nigeria bore the full cost of their breast cancer care. Although a greater proportion of patients in Kenya received financial support for their care, this did not discernibly impact access to healthcare resource utilisation. However, in data pooled across the three countries, a trend towards increased access to innovative targeted therapies (eg, systemic trastuzumab) in all settings for patients who had financial support was observed.

Although this study did not systematically capture breast cancer subtypes, treatment outcomes or the proportion of financial support available to individual patients for specific procedures because of inherent limitations in how data were recorded, the summary analysis of available indices indicates clear deficiencies in specific aspects of care, such as a prolonged turnaround time for core needle biopsy and pathology. The delay in performing core needle biopsy and pathology limits both the ability to quickly and accurately diagnose disease subtype and 
Patients in whom procedure was done, $\%$

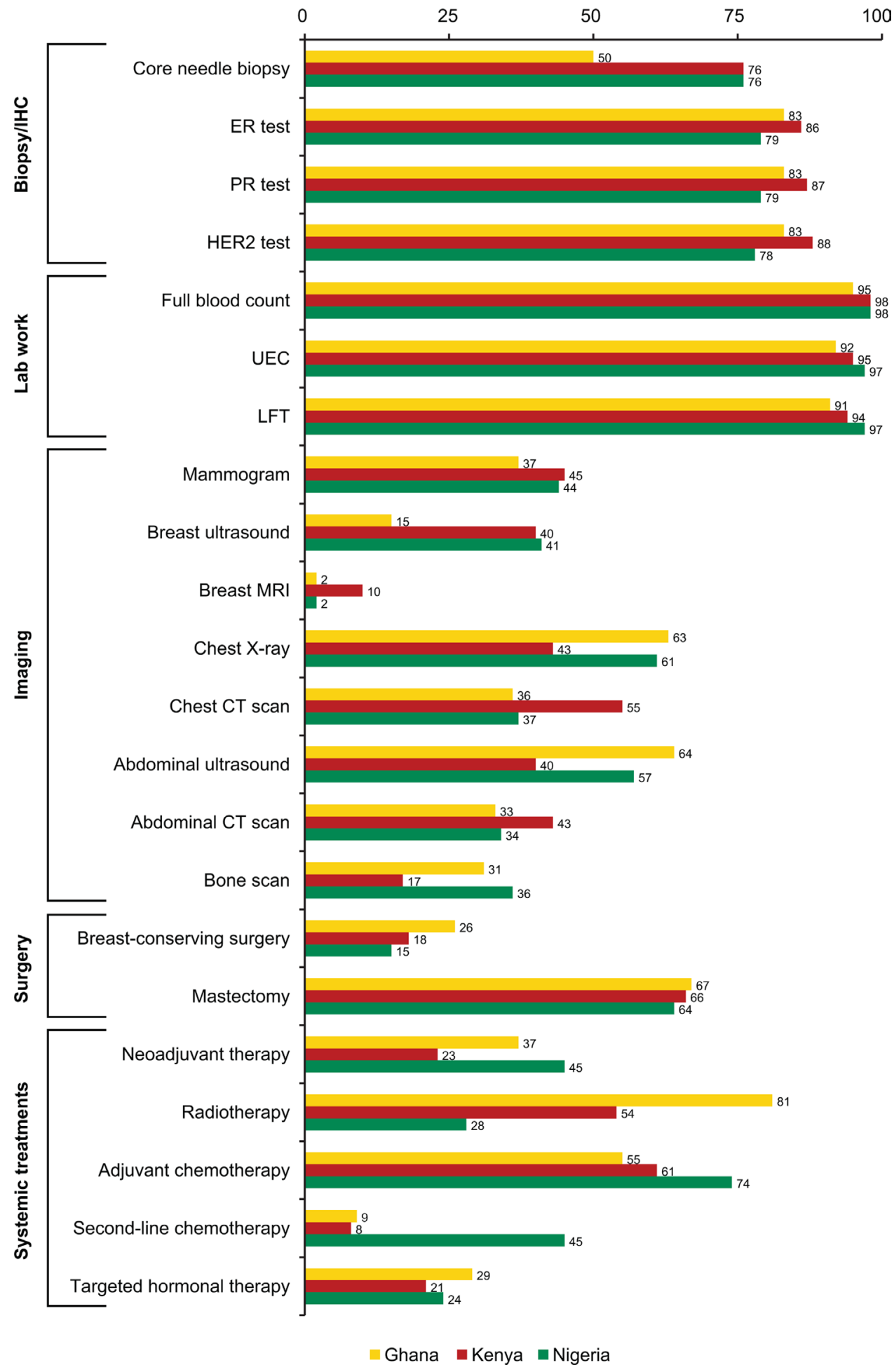

Figure 1 Proportion of patients using key healthcare resources for management of breast cancer. ER, oestrogen receptor; HER2, human epidermal growth factor receptor 2; IHC, immunohistochemistry; LFT, liver function test; PR, progesterone receptor; UEC, urea/electrolytes/creatinine panel.

the timely administration of treatment. Thus, investments to improve the comprehensiveness of services in tertiary facilities and/or to improve the referral systems between cancer services are necessary steps to provide better patient outcomes. Likewise, there are limitations in the availability and affordability of therapies as patients proceed along the treatment journey. As noted, adding radiation facilities in Nigeria would greatly reduce the risk of recurrence in patients with surgical resection and represents a relatively straightforward approach to improve patient outcomes. Across all three countries, the utilisation of systemic therapy for advanced disease 


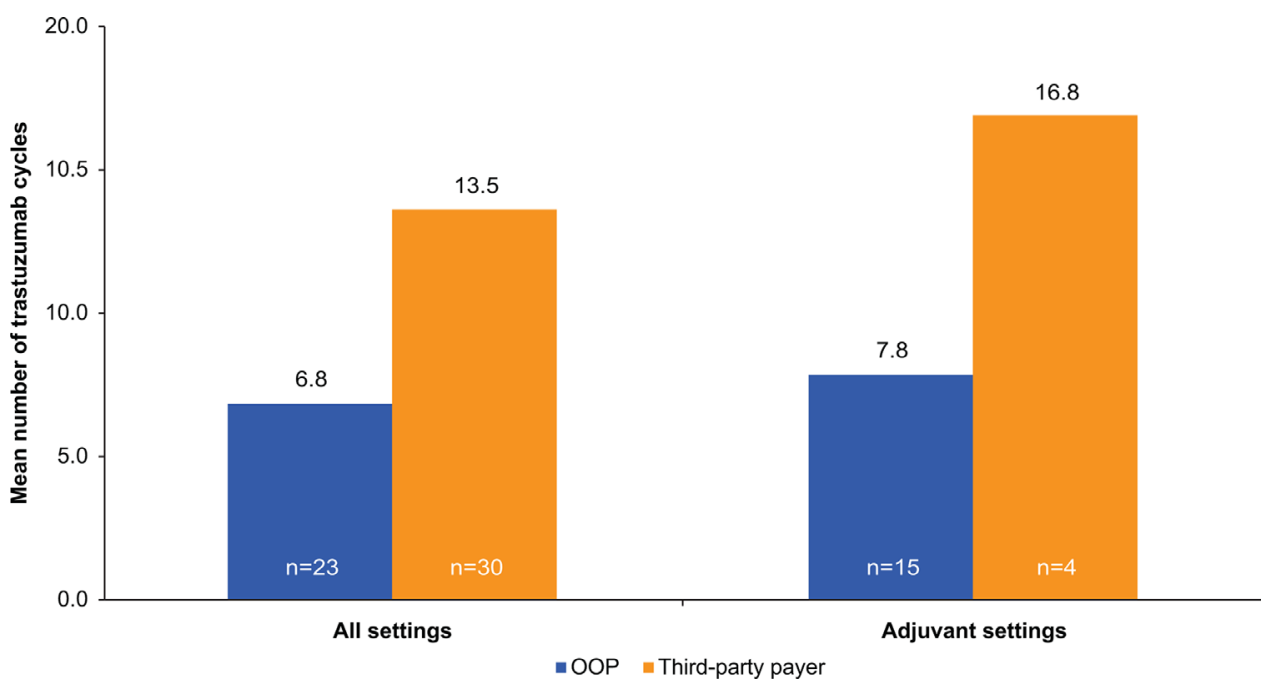

Figure 2 Mean number of cycles of trastuzumab received by patients paying out-of-pocket (OOP) only compared with those with third-party payer support.

was below what should have been expected based on the proportion of patients presenting with advanced disease. Data on the prevalence of various subtypes of breast cancer in African countries are limited, but reports suggest that approximately $50 \%$ of patients have hormone receptor positive disease and approximately $25 \%$ of patients have either HER2-positive disease or triple-negative breast cancer. ${ }^{16}{ }^{17}$ For example, the use of newer generation hormonal agents, such as aromatase inhibitors, was low $(21 \%-29 \%)$, considering that approximately half of the patients would be expected to be hormone receptor positive. ${ }^{16}$ In addition, given that approximately $15 \%-22 \%$ of breast cancers are observed to be HER2 positive, ${ }^{16}$ a lowerthan-expected proportion of patients received HER2targeted therapy $(5 \%-8 \%)$; this is suggestive of poor/ absent healthcare insurance coverage. Furthermore, among 226 evaluable patients in this study, the prevalence of triple-negative breast cancer was $34 \%$ (77/226), luminal A breast cancer was 38\% (83/226), luminal B breast cancer was $5 \%(13 / 226)$ and HER2-positive breast cancer was $22 \%(49 / 226)$. Therefore, it is reasonable to conclude that the use of aromatase inhibitors and trastuzumab in this study was lower than what may be expected based on the prevalence data. In addition, these data show that most patients in Ghana and Nigeria paid for their treatments OOP. Even in Kenya, a significant proportion of patients paid for their procedures OOP. Furthermore, most patients treated at these centres were employed and had received secondary or postsecondary education. Together, these findings suggest that there is limited healthcare insurance coverage to support breast cancer treatment and that the general population may not be aware of healthcare access. Therefore, the governments of Ghana, Kenya and Nigeria need to implement policies regarding breast cancer that will expand healthcare resources and access to healthcare coverage, develop an outreach programme to educate the general population

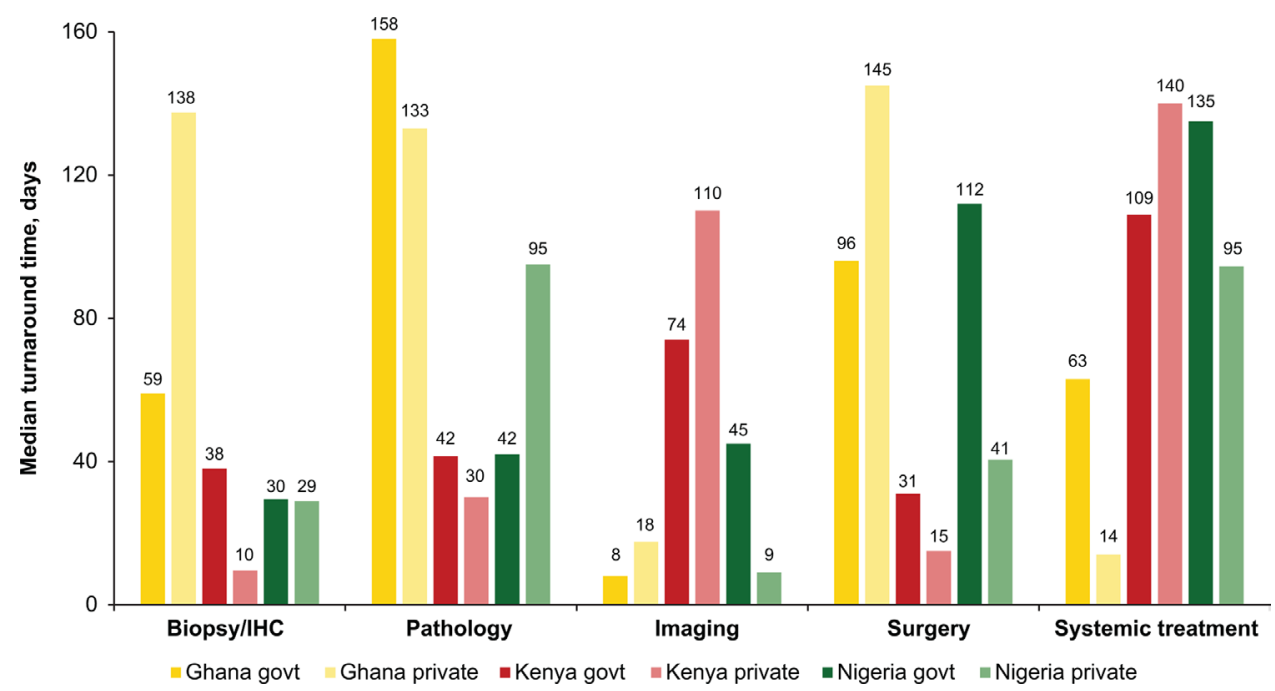

Figure 3 Median turnaround time to receipt of key aspects of care by country and type of tertiary care centre (government vs private). IHC, immunohistochemistry. 
Proportion of patients paying OOP, \%

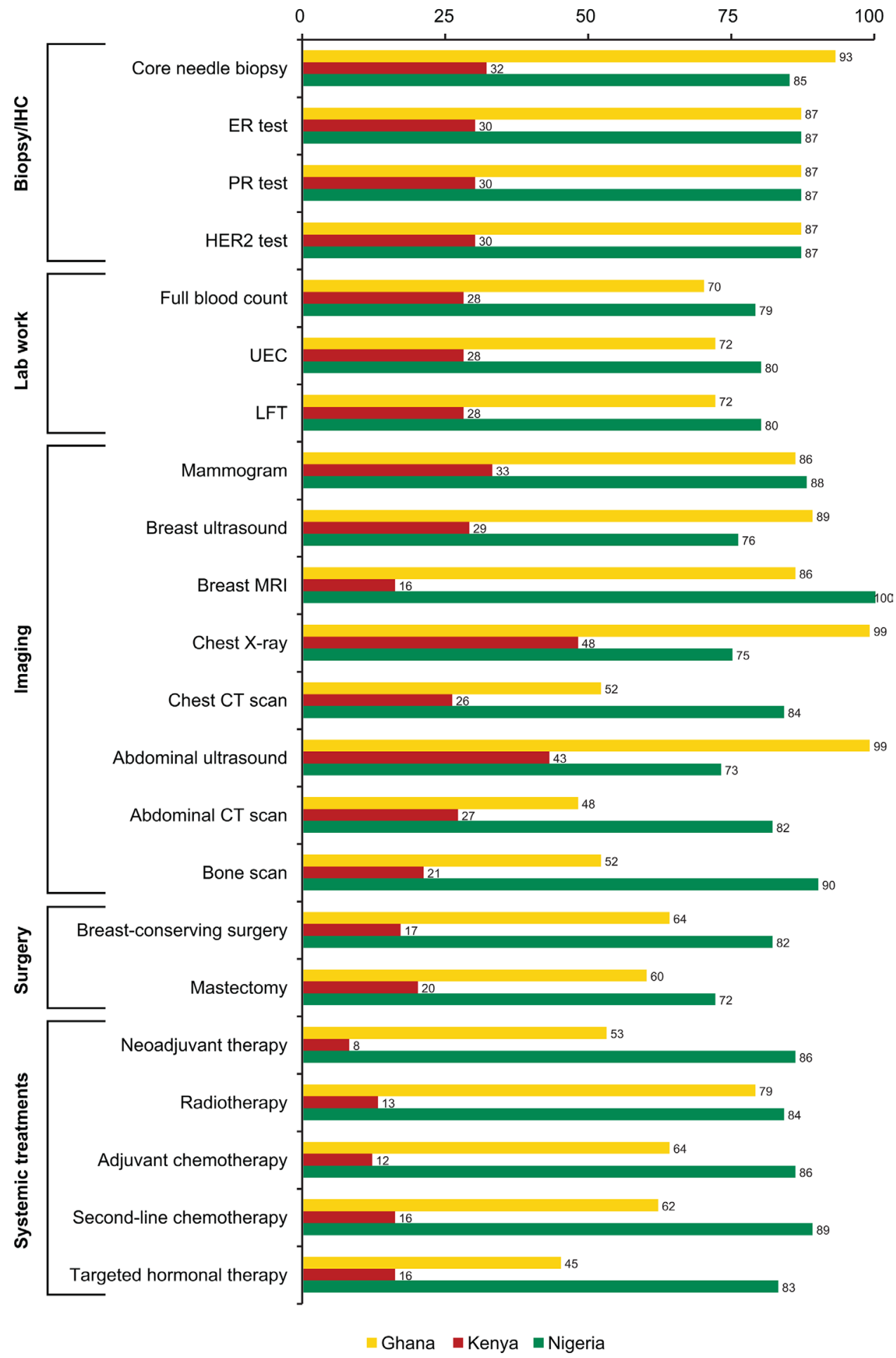

Figure 4 Patients paying OOP for procedures. ER, oestrogen receptor; HER2, human epidermal growth factor receptor 2; IHC, immunohistochemistry; LFT, liver function test; OOP, out of pocket; PR, progesterone receptor; UEC, urea/electrolytes/creatinine panel.

and facilitate awareness of and access to resources and treatment.

The focus of this manuscript is on tertiary care centres that provide the most comprehensive cancer care service rather than primary healthcare services. Thus, the study had the inherent limitation of selection bias towards patients who would seek treatment for their breast cancer at these centres. In addition, the patient charts did not routinely capture information on procedures/diagnoses that may have been performed at other centres but were relevant to the current management plan of the patient. Nonetheless, the data collected for this study suggest that although healthcare 
professionals are likely to recommend standard treatment options to their patients, access to diagnostics services and treatment modalities is delayed and/or inadequate and, in most cases, presents a substantive financial burden to the patient and their caregivers. This is particularly true for situations where advanced diagnostics such as immunohistochemistry and innovative treatments like trastuzumab are required; the documented use of these procedures and treatments is low and is, in part, due to difficulty in paying for these OOP. In this regard, it is noted that private-public partnerships may play a vital role in improving access and affordability. In Ghana for instance, such a partnership that provides immunohistochemistry testing and trastuzumab treatment at no cost to patients at the point of care has greatly increased the diagnosis of, the correct treatment of, and adherence in patients with breast cancer.

Taken together, access and affordability to breast cancer screening, diagnosis and treatment need to be improved through informed and prioritised steps to maximise the impact of investments in healthcare and health reforms in sub-Saharan African countries in general. Additional analyses aimed at evaluating treatment patterns and costs using these records supplemented with other economic data are planned to provide more detailed insights into the challenges and potential for improvement of breast cancer management in sub-Saharan Africa.

\author{
Author affiliations \\ ${ }^{1}$ Aga Khan University - Kenya, Nairobi, Kenya \\ ${ }^{2}$ National Hospital, Abuja, Nigeria \\ ${ }^{3}$ Korle Bu Teaching Hospital, Accra, Ghana \\ ${ }^{4}$ Kenyatta National Hospital, Nairobi, Kenya \\ ${ }^{5}$ Sweden Ghana Medical Centre, Greater Accra, Ghana \\ ${ }^{6}$ Lakeshore Cancer Center, Victoria Island, Lagos, Nigeria \\ ${ }^{7}$ Roche Kenya Ltd, Nairobi, Kenya \\ ${ }^{8}$ Roche Products Ghana Ltd, Accra, Ghana \\ ${ }^{9}$ F. Hoffmann-La Roche Ltd, Kaiseraugst, Switzerland \\ ${ }^{10} \mathrm{~F}$. Hoffmann-La Roche Ltd, Basel, Switzerland
}

Contributors MT, RO, JY, AG, ChuN, CE, GM and EB were involved in data collection. GM, EB, BM and ChaN curated and analysed the study data. All authors contributed to the study design and manuscript writing, and all authors approved the final draft of the manuscript for submission.

Funding The study was funded by F. Hoffmann-La Roche Ltd. Medical editorial support was provided by Jerome Sah, PhD (ApotheCom, San Francisco, California, USA), and funded by F. Hoffmann-La Roche Ltd.

Competing interests MT, RO, AG and CE report no conflicts of interest. JY reports honoraria from F. Hoffmann-La Roche Ltd. ChuN is an employee of and shareholder in Lakeshore Cancer Centre. GM, PA, EB, BM and ChaN are employees of F. Hoffmann-La Roche Ltd.

Patient and public involvement Patients and/or the public were not involved in the design, or conduct, or reporting, or dissemination plans of this research.

Patient consent for publication Not required.

Ethics approval All institutional review board approvals as well as ethical reviews were done in compliance with local and national guidelines and approvals obtained. Institutional review board approvals are available online (http://bit.ly/BCPJ-IRB).

Provenance and peer review Not commissioned; externally peer reviewed.

Data availability statement Data are available on reasonable request. Qualified researchers may request access to individual patient-level data through the clinical study data request platform (www.clinicalstudydatarequest.com). Additional details on Roche's criteria for eligible studies are available here: https://clinicalstudydat
arequest.com/Study-Sponsors/Study-Sponsors-Roche.aspx. For more details on Roche's Global Policy on the Sharing of Clinical Information and how to request access to related clinical study documents, click here: https://www.roche.com/ research_and_development/who_we_are_how_we_work/clinical_trials/our_ commitment_to_data_sharing.htm.

Open access This is an open access article distributed in accordance with the Creative Commons Attribution Non Commercial (CC BY-NC 4.0) license, which permits others to distribute, remix, adapt, build upon this work non-commercially, and license their derivative works on different terms, provided the original work is properly cited, appropriate credit is given, any changes made indicated, and the use is non-commercial. See: http://creativecommons.org/licenses/by-nc/4.0/.

ORCID iD

Majid Twahir http://orcid.org/0000-0002-0414-2925

\section{REFERENCES}

1 Bray F, Ferlay J, Soerjomataram I, et al. Global cancer statistics 2018: GLOBOCAN estimates of incidence and mortality worldwide for 36 cancers in 185 countries. CA Cancer J Clin 2018;68:394-424.

2 Adeloye D, Sowunmi OY, Jacobs W, et al. Estimating the incidence of breast cancer in Africa: a systematic review and meta-analysis. $J$ Glob Health 2018;8:010419.

3 , Fitzmaurice C, Akinyemiju TF, et al, Global Burden of Disease Cancer Collaboration. Global, regional, and National cancer incidence, mortality, years of life lost, years lived with disability, and Disability-Adjusted life-years for 29 cancer groups, 1990 to 2016: a systematic analysis for the global burden of disease study. JAMA Oncol 2018;4:1553-68.

4 Akuoko CP, Armah E, Sarpong T, et al. Barriers to early presentation and diagnosis of breast cancer among African women living in subSaharan Africa. PLoS One 2017;12:e0171024.

5 Espina C, McKenzie F, Dos-Santos-Silva I. Delayed presentation and diagnosis of breast cancer in African women: a systematic review. Ann Epidemiol 2017;27:e657:659-71.

6 Azubuike SO, Muirhead C, Hayes L, et al. Rising global burden of breast cancer: the case of sub-Saharan Africa (with emphasis on Nigeria) and implications for regional development: a review. World $J$ Surg Oncol 2018;16:63.

7 Akarolo-Anthony SN, Ogundiran TO, Adebamowo CA. Emerging breast cancer epidemic: evidence from Africa. Breast Cancer Res 2010;12:S8.

8 Edge SB, Compton CC. The American joint Committee on cancer: the 7th edition of the AJCC cancer staging manual and the future of TNM. Ann Surg Oncol 2010;17:1471-4.

9 Vlahiotis A, Griffin B, Stavros AT, et al. Analysis of utilization patterns and associated costs of the breast imaging and diagnostic procedures after screening mammography. Clinicoecon Outcomes Res 2018;10:157-67.

10 Black E, Richmond R. Improving early detection of breast cancer in sub-Saharan Africa: why mammography may not be the way forward. Global Health 2019;15:3.

11 Cho K, Tyldesley S, Speers C. The utilization and impact of core needle biopsy diagnosis on breast cancer outcomes in British Columbia. Br Columbia Med J 2014;56.

12 Spitale A, Mazzola P, Soldini D, et al. Breast cancer classification according to immunohistochemical markers: clinicopathologic features and short-term survival analysis in a population-based study from the South of Switzerland. Ann Oncol 2009;20:628-35.

13 Selove R, Kilbourne B, Fadden MK, et al. Time from screening mammography to biopsy and from biopsy to breast cancer treatment among black and white, women Medicare beneficiaries not participating in a health maintenance organization. Womens Health Issues 2016;26:642-7.

14 Oppong BA, Dash C, Coleman T, et al. Time to diagnostic evaluation after mammographic screening in an urban setting. $J$ Womens Health 2016;25:1225-30.

15 Martei YM, Narasimhamurthy M, Prabhakar P, et al. Breast cancer pathology turnaround time in Botswana. J Glob Oncol 2018;4:1-7.

16 Galukande M, Wabinga H, Mirembe F, et al. Molecular breast cancer subtypes prevalence in an Indigenous sub Saharan African population. Pan Afr Med J 2014;17:249.

17 Brandão M, Guisseve A, Bata G, et al. Breast cancer subtypes: implications for the treatment and survival of patients in Africa-a prospective cohort study from Mozambique. ESMO Open 2020;5:e000829. 